\title{
T- JOINTS - EXPERIMENTAL AND NUMERICAL ANALYSIS
}

The article presented focuses on the comparison of the measurement results obtained in laboratory experiments on joints of a lattice girder. Because of the geometric variety of joints, the article presented concentrated selectively on hinged T-joints only. The geometry of this type of joint consists of two fundamental members: the chord and the brace member. The T-joints examined were always composed of the rectangular chord member in combination with a variety of types (rectangular, circular and open) and dimensions of brace members. The scientific research results and evaluations presented characterize the correlations regarding the global resistance of joints in lattice structures in the light of the latest scientific knowledge that should be responsibly taken into consideration in their reliable and cost-effective design.

Keywords: T-joints, circular hollow sections, rectangular hollow sections, HEA-sections, vertical and horizontal deformation.

\section{Introduction}

The gradual increase in the production range of hollow sections has resulted in their more common application in various areas and types of construction. This type of section can find a number of applications in the construction industry, especially in structures where attractive general appearance of the whole structure is required [1].

From the point of view of a structural designer, the application of hollow section joints presents relatively effective utilisation of the material. The distribution of the material and its mass in hollow section joints brings several advantages for various kinds of load. Their application is one of the best design solutions in members under compression, with respect to their buckling, as well as in members loaded in bending, with respect to their lateral-torsional buckling as well.

As far as strain is concerned, joints present the most exposed areas in structures composed of such types of section. Joints composed of rectangular hollow sections have recently received quite close attention. Our experimental research programme concerns the hollow-section joints combined with other kinds of section [2].

The article presents the results of the experimental programme that has been carried out at the Faculty of Civil Engineering of the Technical University in Košice. Attention is paid to the joints composed of rectangular and circular hollow sections, and HEAtype open sections. These are all T-joints where the continuous chord member is made of a rectangular hollow section in each type of specimen. The brace members vary in section in terms of type and dimensions. Due to a large variety of combinations of sections in terms of their type and dimensions, the authors of the article are planning to extend their research and supplement the results gradually by using some other combinations and types of section in the future.

\section{Experimental analysis}

\subsection{Experimental plan}

Hollow sections are applicable particularly in the construction of lattice structures. In the design of such structural members the essential loading component is an axial force - either in tension or in compression. Given these facts can be concluded that the joint in a lattice structure belongs to one of the most exposed areas. When designing the joint itself it is necessary to take into consideration a number of factors having an impact on its stiffness, such as geometrical data, the stiffness and strength ratios between the chord and brace members, and similarly, the slenderness ratios of web sections, material properties and, last but not least, the magnitude of action [4].

On the basis of the mentioned above requirements, an experimental programme was drawn up whose purpose was to verify and supplement the acquired knowledge on the actual behaviour of joints in lattice structures with rectangular hollow sections, also depending on combinations of various types of hollow section and hollow and open sections [4-5].

As the scope of the problem under investigation is so vast, attention was focused on and limited to T-joints. The angle between the chord and brace members was identical in all cases, i.e. $90^{\circ}$. For the chord member of the joint under investigation, the upper compression member of a lattice structure was selected, while the

\footnotetext{
* Vincent Kvocak, Pavol Beke, Renata Vargova

Faculty of Civil Engineering, Technical University of Kosice, Slovakia, E-mail: vincent.kvocak@tuke.sk
} 


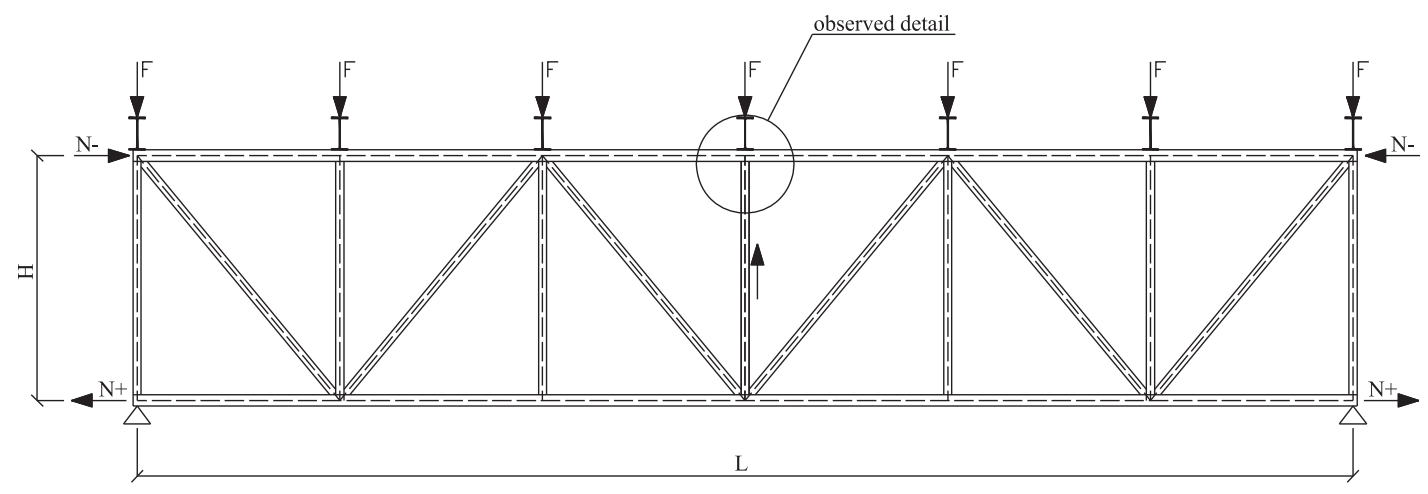

Fig. 1 Arrangement of joint members and their mutual action

brace member was the vertical compression member in a lattice structure. The arrangement of the joint members and their mutual action is shown in the following Fig. 1.

From the geometrical point of view, three types of joint were compared. In the first type, both the chord and brace members were composed of rectangular hollow sections. In the second type of joint, the chord member consisted of a rectangular hollow section, whereas the vertical brace member was made of a circular hollow section. In the third variant of joint, similar to the previous types, the chord member was composed of a rectangular hollow section and the brace member was represented by an open HEA-section. For the purpose of mutual comparison of the results obtained for the individual types of section, the dimensions and the cross-section of the chord member always remained equal [5].

The brace member varied both in size and shape of section. The ratio of the mean diameter or width of the vertical brace member, to that of the horizontal chord is called the $\beta$-parameter. In rectangular and circular sections was applied $\beta=0.43$ (60/140), 0.71 $(100 / 140)$ and $1.0(140 / 140)$ respectively. HEA-sections are an exception where the experimental programme had to be adjusted to the rolling mill plan for such types of section. In this type of joint, $\beta$-parameters were $0.71(100 / 140), 0.86(120 / 140)$ and 1.0 (140/140) respectively. Thus, for rectangular hollow sections, the following sections were selected: RHS $140 \times 140 \times 4$, RHS $100 \times$ $100 \times 3$, RHS $60 \times 60 \times 3$, for circular bracing members: CHS $140 \times 5$, CHS $100 \times 5$, CHS $60 \times 4$ and for open sections: HEA 140, HEA 120, and HEA 100 . The chord member for each type of joint composed of RHS $140 \times 140 \times 4$. The $\beta$-parameter was one of the most significant factors in the comparison and evaluation of the resulting effects of action of joints of circular, rectangular and open rolled-steel HEA sections. The goal of the research was to cover the greatest possible spectrum of $\beta$-parameters [6].

The above parameters made it possible to create a total of nine experimental types of joint (three joints consisting of circular sections, three joints consisting of square sections, and finally, three joints with open brace members). For each test type/set of joints there were subsets of three identical test specimens. Geometry of test specimens and shape of loading test assembly is shown in Fig. 2.
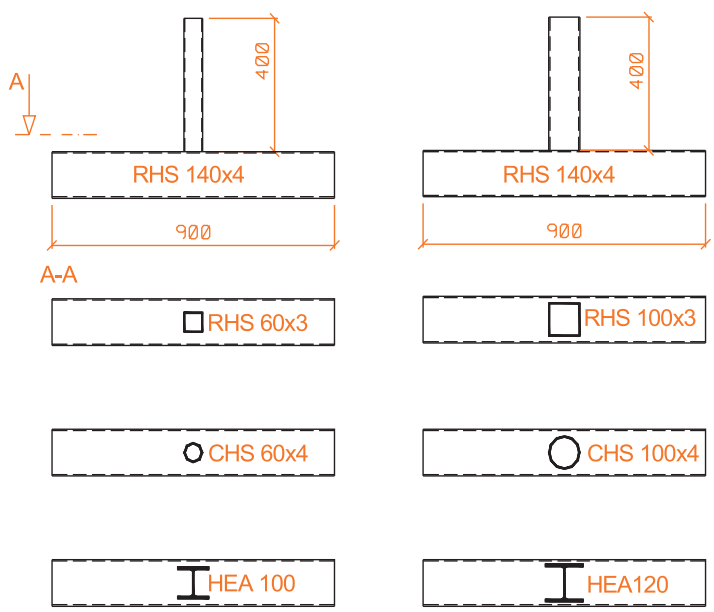
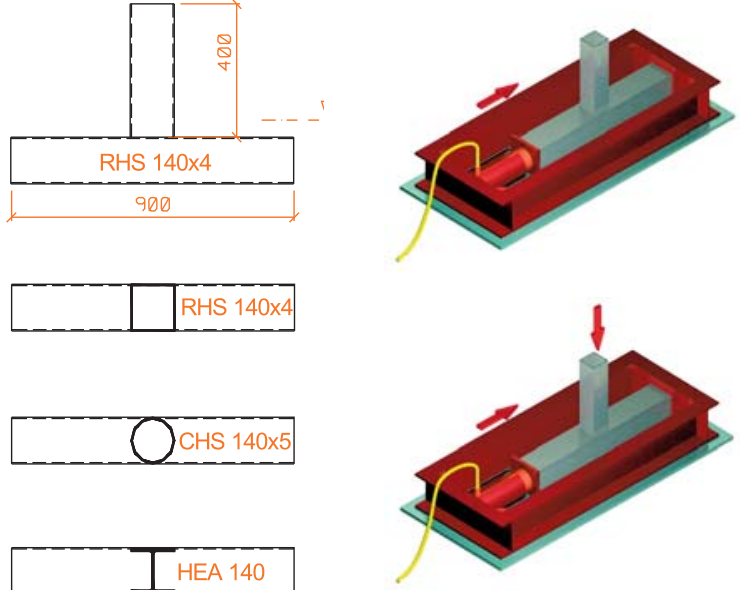

Fig. 2 Geometry of test specimens 
All models were made of steel S235 and a specimen of each model was taken to analyse its material characteristics. The yield strength in all specimens did not exceed the one declared by the steel manufacturer and it varied between 290 and $320 \mathrm{MPa}$.

To simulate the real behaviour of the joint in a lattice structure, the chord member was assumed to act in horizontal compression, while the brace members were compressed in a vertical direction. Horizontal compression was actuated by means of an additional frame and pneumatic press (see Fig. 2). The numerical value of horizontal load remained constant during the whole loading period. Each specimen type was tested at three horizontal force levels: $68 \mathrm{kN}, 115 \mathrm{kN}$ and $192 \mathrm{kN}$. Vertical load imposed on the specimens by the main hydraulic press was gradually increased until the total failure of the specimen. With respect to the complexity of the task, the length of the brace member was designed so as to prevent buckling of the member.

Measurement points on the individual specimens were then selected. Strain gauges were used to measure stress in the specimens and inductive sensors to determine horizontal and vertical deformations. During the strain-gauge measurements attention was focused on the chord member as, based on the calculation model, the joints under investigation collapsed due to the failure of the chord member in particular. Stresses were examined both in the horizontal and vertical webs of the chord and the strain gauges were attached in both longitudinal and transverse directions. Arrangement of strain gauges is shown in Fig. 3a. As can be seen from Fig. $3 \mathrm{~b}$, deformation was measured mainly in the chord members, specifically in the upper horizontal and vertical webs of the chord member section. tions and to this end - to simulate such conditions - the above mentioned software seemed to be the most suitable.

The process of constructing a theoretical model with the aid of the mentioned above software package consists of several steps. The external CAD description of the structure's geometric features is fundamental to the construction of such a model. The structure's geometry was imported into the Ansys software package by means of an external pre-existing SAT file. Once the finite element geometric model was created and checked, the joint to be analysed was divided into finite elements. After generating and refining the mesh network in the model, the structural simulation module was activated. Here, the simulation module serves the purpose of determining the type of computation and thus imposing proper boundary conditions - support and loading constraints. In the joint analysis a static structural analysis module was applied.

The most complex issue in the construction of a theoretical model is the proper specification of constraints. The constraints were imposed with special attention paid to the real behaviour of the joint in the course of the experiment. The correctness of the support constraints was verified by comparison with the theoretical and real deformation curves.

The simulation of loading conditions was compatible with the experimental measurements, and the load was applied on both chord and brace members. The horizontal load was considered to be constant, while the vertical load was gradually increased.

Material non-linearity was taken into account by using Young's modulus with linear strain hardening in the model. The yield strength

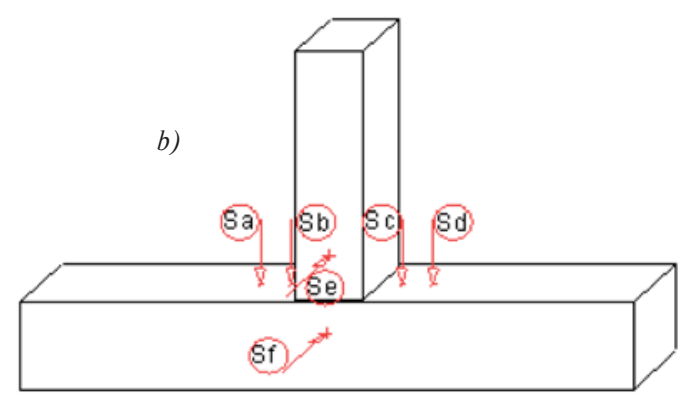

Fig. 3 Arrangement of (a) strain gauges, (b) inductive sensors and deformeters

\subsection{Theoretical Finite Element Analysis model}

For the purpose of verifying and confirming the experimental measurements theoretical models of the joints under investigation were constructed using the software package Ansys 11, specifically its graphical upgrade Workbench 11 [7-8].

In order to obtain the most credible results, it was necessary to consider both geometric and material non-linearity in the calcula- corresponded to its true value that was obtained in tensile tests. Geometric non-linearity was taken into consideration by activating the function of large deflections.

After the input of all necessary characteristics the computation itself was conducted. The evaluation of the joint was carried out on the basis of deformations/strains or stresses. Considering the fact that the stresses (strains) could not be compared with the 
experiment in a precise manner, the deformations of the joint were essential for the comparison. Although it was not possible to pinpoint precisely where strains were evaluated in the experimental measurement, the theoretical stress analysis provides an overall view of the joint behaviour. From the stress-related point of view, it made possible the evaluation of the points that could not be included for practical reasons, especially those on the interior webs of the chord member. In conclusion, the construction of the theoretical model confirmed the experimental results and facilitated the analysis of other joints.

\section{Obtained results}

The types of joints selected were observed for both stress and deformation. In the following section of the article, attention will be directed to the deformation of the joints. The following sections provide an exact view of the real behaviour of the individual types of joint. The figures presented compare both vertical and horizontal experimentally measured deformations of the joints.

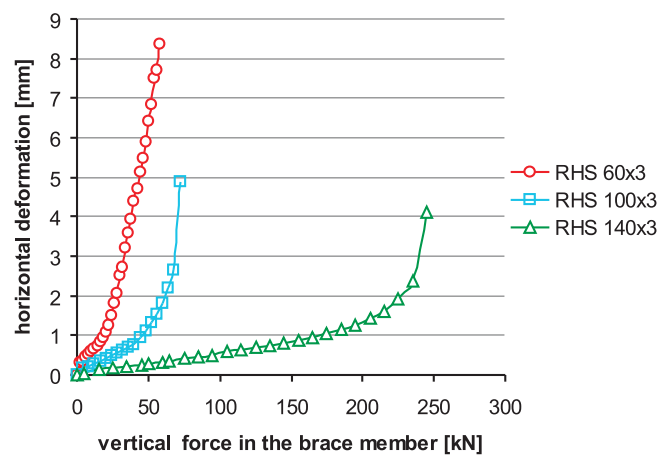

Fig. 4 Comparison of the experimentally measured horizontal and vertical deformations in the joints composed of the brace members with rectangular hollow sections
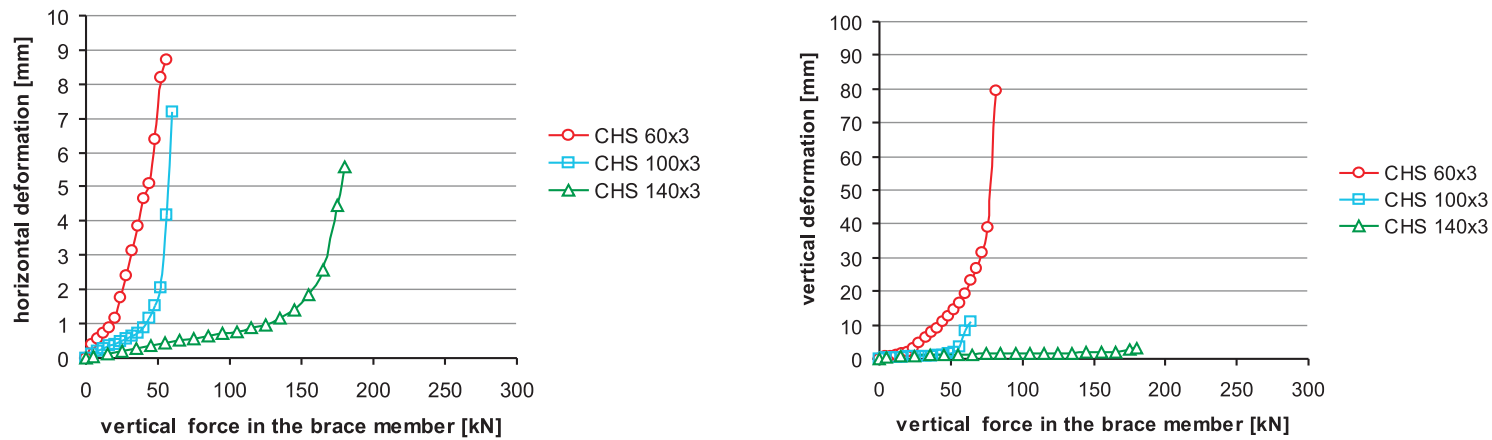

Fig. 5 Comparison of the experimentally measured horizontal and vertical deformations in the joints composed of the brace members with circular hollow sections

\subsection{Comparison of deformations in the joints consisting of the identical type of the brace member}

The joints with the identical type of the brace member are compared (Fig. 4 - RHS sections, Fig. 5 - CHS sections and Fig. 6 HEA sections). First, when there is linear deformation, the joint is in the elastic range. Later, as the load is increased, the joints deviate from this linear proportionality and the deformation goes through the elasto-plastic and eventually into its plastic range of action.

Of all the types of brace members, the most resistant certainly seem to be those with $b_{0}=b_{1}(\beta=1)$. From the deformation point of view, the joints with a width of $60 \mathrm{~mm}$ are the least suitable. These exhibited excessive deformations even under minimal load.

\subsection{Comparison of deformations in the joints with the same widths of chord and brace members}

The stiffness of the joint does not depend only on its dimensions but also on the type of the brace member used. The comparisons are presented in the form of the following Figs. 7-9.

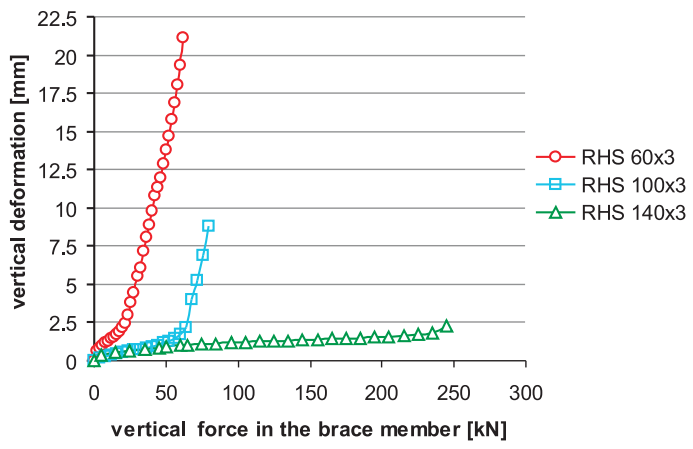



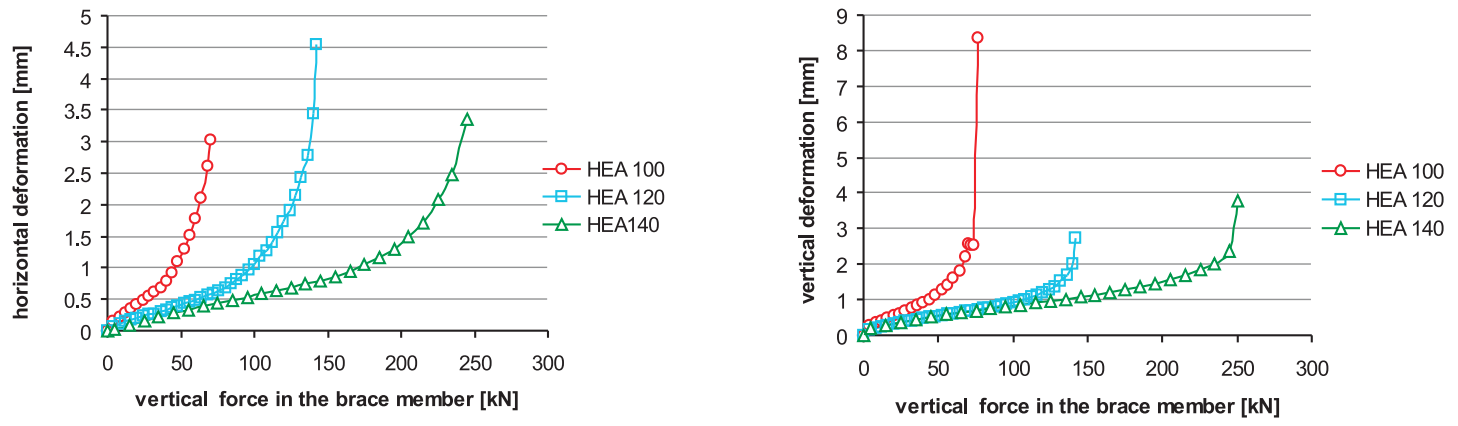

Fig. 6 Comparison of the experimentally measured horizontal and vertical deformations in the joints composed of the brace members with open HEA-sections

In the first type of joint $(\beta=1)$, the stability of the chord web was crucial for the overall resistance of the joint. Figure 7 presents the distribution of values of vertical and horizontal deformation. Significant deformation of the vertical chord web occurred even under a relatively light vertical load. The joint collapsed completely with the gradual increase in load due to the buckling of the vertical web of the horizontal chord member. When the resistance of rectangular, circular and open sections was compared, the rectangular sections proved to be the stiffest.

As can be seen from Fig. 8, the buckling effect of the chord web on the overall resistance of the joint can be observed also in the joints with $\beta=0.714$. The overall resistance of the joint was influenced by the loss of stability of the vertical web although the difference between the vertical and horizontal deformation was less significant than in the first case. When comparing the types of brace member used, the rectangular section appeared to be the most resistant of all. However, the difference between the rectangular and open HEA-section was minimal regarding vertical and horizontal deformations.

In the third type of joint (Fig. 9) with the most slender brace members, the overall resistance of the joint was to a great extent affected by the stiffness of the horizontal chord web. The difference
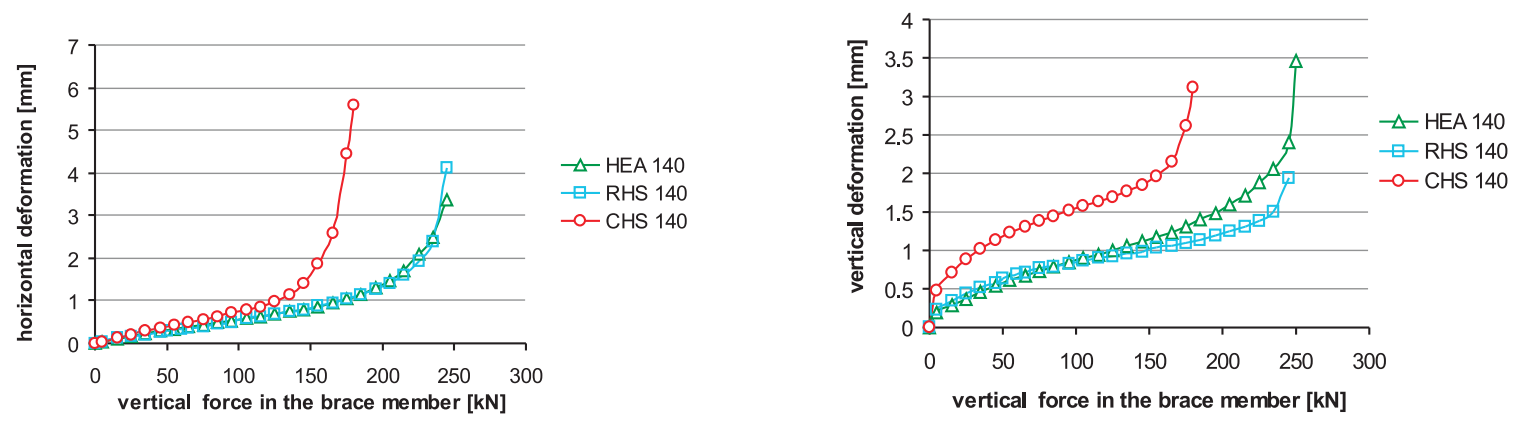

Fig. 7 Horizontal and vertical deformation of the T-joint with $\beta=1.00$ depending on the type of brace member used
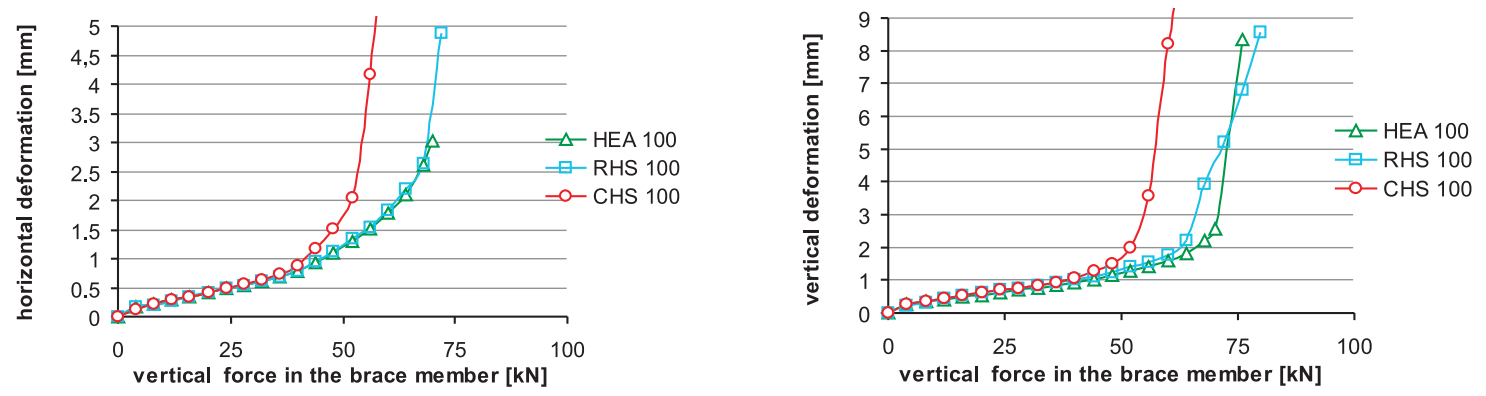

Fig. 8 Horizontal and vertical deformation of the T-joint with $\beta=0.714$ depending on the type of brace member used 


\section{COMMNICOIIONS}
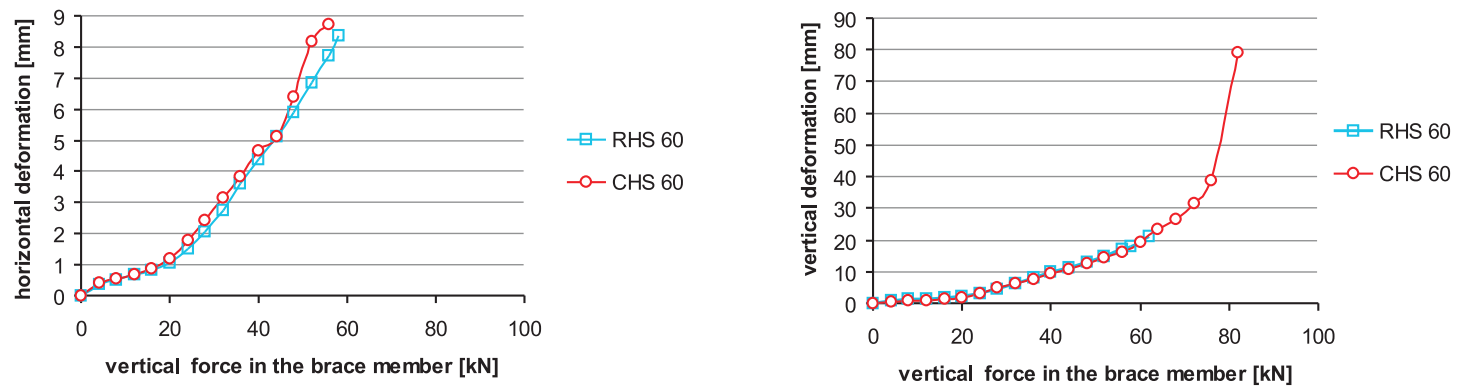

Fig. 9 Horizontal and vertical deformation of the T-joint with $\beta=0.428$ depending on the type of brace member used

between the horizontal and vertical deformation in this type of joint is the biggest and the vertical deformation the greatest. The influence of the type of the brace member used on the overall resistance of such joints is virtually negligible. Due to the limited possibilities of the HEA rolling programme, only circular and rectangular hollow sections were compared. For $\beta=0.714$ the behaviour of open sections was identical to that of rectangular sections and, obviously, it does not change for lower $\beta$-parameters.

\section{Resistances of the individual types of joints}

Two essential loading states were recorded during the entire loading cycle: one represented the resistance of the joint and the other at which the joint failed.

The resistance of the joint is the loading point at which, without increasing the load, deformation continues to rise. This point is the state when the joint departs from its elastic range and gradually goes from the elasto-plastic into the plastic range of behaviour. The resistances of the joints in horizontal compression in the chord of $192 \mathrm{kN}$ are given in Table 1.

The table above compares the experimental values of resistances with the resistances determined using different calculation techniques. For the determination of the experimental resistances the joints with the most subtle brace members seemed to be most problematic. With this type of joint, it was impossible during the loading cycle to specify the load that would correspond to the resistance of the joint. No plastic flow occurred even during the heaviest vertical load, nevertheless, the joints exhibited excessive deformations even during the smallest vertical loads. Therefore, the resistance was determined on the basis of the limit of deformation (Table 2). The vertical deformation of $1.5 \mathrm{~mm}$ that occurred at the vertical load of $20 \mathrm{kN}$ was considered as the limit of deformation. The limit deformation of $1.50 \mathrm{~mm}$ corresponds to measured points $\mathrm{Sb}$ and Sc (see Fig. 3). The given resistance corresponds to the joints with both rectangular and circular brace member sections.

\begin{tabular}{|c|c|c|c|c|c|}
\hline & \multicolumn{5}{|c|}{ Joint resistances $[\mathrm{kN}]$} \\
\hline & Experiment & STN EN 1993-1- 8 & ANSYS & Exp/STN EN & Exp/Ansys \\
\hline RHS 60 & 21 & 21 & 19 & 1.00 & 1.11 \\
\hline RHS 80 & - & 35 & 30 & - & - \\
\hline RHS100 & 56 & 54 & 55 & 0.96 & 1.02 \\
\hline RHS120 & - & 146 & 141 & - & - \\
\hline RHS140 & 170 & 165 & 167 & 0.97 & 1.02 \\
\hline CHS 60 & 21 & 16 & 20 & 0.80 & 1.00 \\
\hline CHS 80 & - & 27 & 26 & - & - \\
\hline CHS 100 & 49 & 42 & 46 & 0.86 & 1.07 \\
\hline CHS120 & - & 109 & 109 & - & - \\
\hline CHS140 & 130 & 129 & 120 & 0.99 & 1.08 \\
\hline HEA 60 & - & - & 20 & - & - \\
\hline HEA 80 & & - & 34 & - & - \\
\hline HEA 100 & 42 & - & 44 & - & 0.95 \\
\hline HEA 120 & 101 & - & 102 & - & 0.99 \\
\hline HEA 140 & 191 & - & 195 & - & 0.98 \\
\hline
\end{tabular}


Resistances of the individual specimen types together with the horizontal and vertical deformations at the ultimate limit state

\begin{tabular}{|c|c|c|c|}
\hline $\begin{array}{c}\text { Brace member } \\
\text { section }\end{array}$ & $\begin{array}{c}\text { Experiment } \\
\text { resistance } \\
{[\mathrm{kN}]}\end{array}$ & $\begin{array}{c}\text { Vertical } \\
\text { deformation } \\
{[\mathrm{mm}]}\end{array}$ & $\begin{array}{c}\text { Horizontal } \\
\text { deformation } \\
{[\mathrm{mm}]}\end{array}$ \\
\hline RHS $60 \times 3$ & 21 & 1.50 & 0.74 \\
\hline RHS $100 \times 3$ & 56 & 1.29 & 1.39 \\
\hline RHS $140 \times 4$ & 170 & 0.59 & 0.91 \\
\hline CHS $60 \times 4$ & 20 & 1.51 & 0.86 \\
\hline CHS $100 \times 4$ & 49 & 1.47 & 1.69 \\
\hline CHS $140 \times 5$ & 130 & 0.89 & 1.04 \\
\hline HEA100 & 42 & 0.72 & 0.72 \\
\hline HEA120 & 101 & 0.82 & 1.00 \\
\hline HEA140 & 191 & 1.06 & 1.24 \\
\hline
\end{tabular}

In the determination of the design resistance values, according to the standards, the resistances of the joints with open HEA brace member sections are omitted. The relevant standard, on the

Table 2. basis of which the resistances were determined, makes provisions for such types of joint but takes a different joint geometry into account (providing that the brace member is rotated by $90^{\circ}$ ).

The following Fig. 10 represents the resistances for the individual types of joint. The values obtained in the experimental measurements, those determined in compliance with the standard requirements set by STN EN 1993-1-8 [9], those determined using the FEA computation models are compared.

\section{Conclusion}

The article presented points to the significance and actuality of the issue of investigating the joints in lattice structures. The priority of the research was to acquire knowledge of the real behaviour of the joints and supplement it with more effective methods for the design of joints composed of rectangular and circular hollow sections, and open HEA-sections. Because of the geometric variety of joints, the work presented concentrated selectively on hinged Tjoints only. T-joints along with other basic uniplanar joints create basic members in lattice structures. The geometry of this type of joint consists of two fundamental members: the chord and the brace member. The T-joints examined were always composed of the rectangular chord member in combination with a variety of types (rectangular, circular and open) and dimensions of brace members.

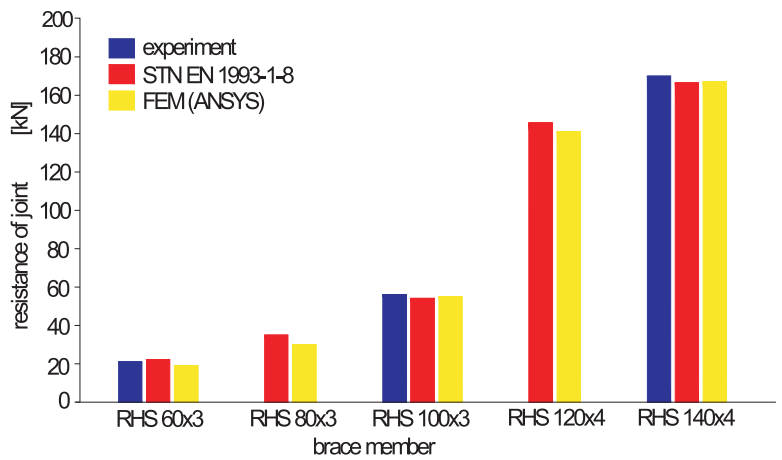

a)

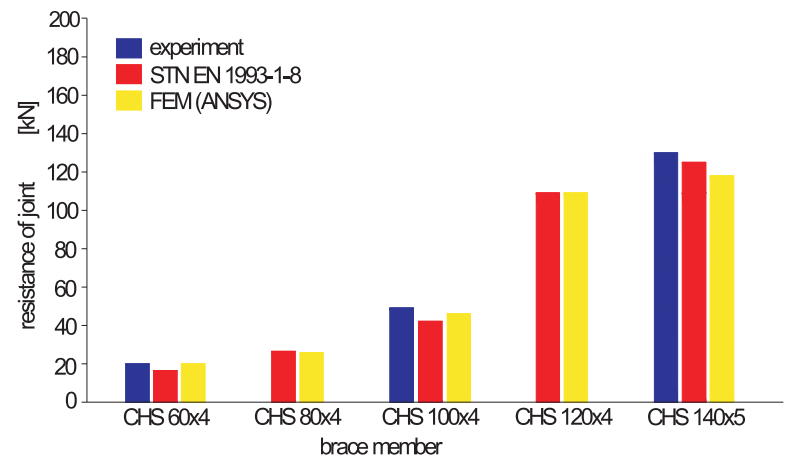

b)

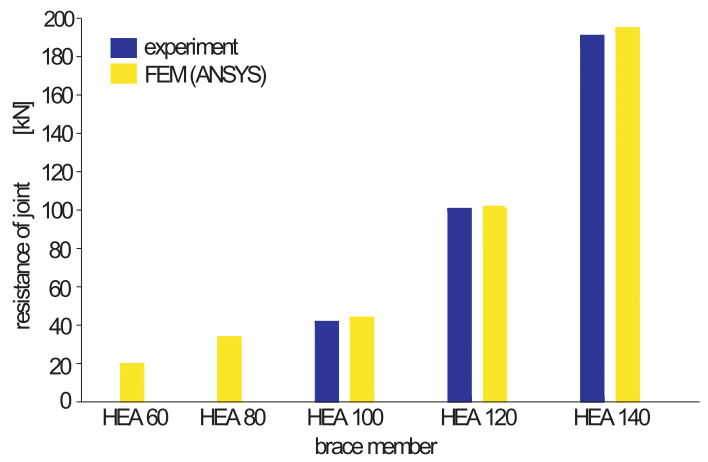

c)

Fig. 10 Resistances comparison of the joints composed of a) rectangular hollow section brace b) circular hollow section and c) open HEA-section brace members 
From the results obtained some patterns of behaviour of Tjoints may be identified. With respect to the geometry and type of section, it can be concluded that the resistance of a joint with $\beta=$ $=1.00$ is greatly influenced by the type of brace member. This influence sharply diminishes with the decreasing value of a $\beta$-parameter. With very low $\beta$-parameters, the influence of the type of brace member becomes virtually negligible and unimportant.

The application of joints composed of hollow sections is encouraged in the standards for the design of steel structures currently in force. The standards; however, make no provisions for combinations of hollow and open sections. The research presented in the article underlines the need for the re-evaluation of the criteria set in the current standards. These should be supplemented with new perfectly legitimate normative requirements for the resistances of the above-mentioned joints according to the presented results. Nowadays authors prepared the analysis of such joints, while the main emphasis is placed on the verification of the obtained results using an appropriate finite model for the joints in question.

\section{Acknowledgment}

This research has been carried out in terms of the project NFP 26220120037 "Centre of excellent research of the progressive building structures, materials and technologies", supported from the European Union Structural funds and Grant VEGA No. 1/0788/12: "Theoretical and Experimental Analysis of Stability and Strength of Composite Members in Compression and Bending" of the grant agency VEGA of the Ministry of Education of the Slovak Republic and the Slovak Academy of Science.

\section{References}

[1] PACKER, J.A., WARDENIER J., KUROBANE Y., DUTTA D., YEOMANS N.: Design Guide for Rectangular Hollow Section (RHS) Joints under Predominantly Static Loading. Verlag TUV Rheiland

[2] RADIC, I., MARKULAK, D., MIKOLIN, M.: Design and FEM Modelling of Steel Truss Girder Joints. Strojarstvo, vol. 52, ISSN 0562-1887, pp.125-135

[3] NIKOLIC, R., VELJKOVIC, J., VICAN, J.: Design of Columns Centrically Loaded in Compression. Communications - Scientific Letters of the University of Zilina. vol. 9, No. 3, 2007, ISSN 1335-4205, pp. 51-55

[4] WARDENIER, J., KUROBANE Y., PACKER J.A., DUTTA D., YEOMANS N.: Design Guide for Circular Hollow Section (CHS) Joints under Predominantly Static Loading. Verlag TUV Rheiland

[5] BEKE, P.: Analyza zvaranych stycnikov z pravouhlych dutych prierezov [Analysis of Welded Joint Composed of Rectangular Hollow Section]. Dissertation work, Kosice, 2009, 135 p.

[6] BEKE, P.; KVOCAK, V.: Analysis of Joints Created from Various Types of Sections. Proc. from $5^{\text {th }}$ European Conference on Steel and Composite Structures, Eurosteel 2008 Graz, Brussels- ECCS, ISBN 92-0147-000-90, pp. 531-536

[7] FREITAS, A. M. S., MENDES, F.C., FREITAS, M. S. R.: Finite Elements Analyses of Welded T-Joints. Proc. from 5th European Conference on Steel and Composite Structures, Eurosteel 2008, Graz, Brussels- ECCS, ISBN 92-0147-000-90, pp. 555-560

[8] VICAN, J., SYKORA, M.: Design of Compression Members for Durability. Communications - Scientific Letters of the University of Zilina. vol. 11, No. 4/2009, ISSN 1335-4205, pp. 9-14

[9] EN 1993-1-8 Eurocode 3: Design of Steel Construction. Part 1-8 Design of joints. 